\title{
Rapid HLA typing by multiplex amplification refractory mutation system
}

\author{
P Patel, Y-M D Lo, J I Bell, J S Wainscoat
}

\begin{abstract}
Aims-To detect HLA susceptibility and protective alleles associated with insulin dependent diabetes mellitus (IDDM) using a multiplex amplification refractory mutation system (ARMS). These include DR3 and DR4 alleles at the DRB1 locus, presence or absence of aspartic acid at position 57 (Asp-57) of the DQB1 locus, and presence or absence of arginine at position 52 (Arg-52) of the DQA1 locus.

Methods-The ARMS approach was used to design allele specific primers for the detection of the major susceptibility and protective alleles for IDDM. These include DR3 and DR4 alleles at the DRB1 locus, Asp-57 and non-Asp-57 at the DQB1 locus, and Arg-52 and nonArg-52 alleles at the DQA1 locus. The allele specificity of each set of primers was first tested separately using DNA samples from 15 individuals previously typed for the DRB1, DQB1, and DQA1 loci using the sequence specific oligonucleotide (SSO) technique. The possibility of using multiplex ARMS for typing multiple susceptibility/protective alleles for IDDM was further investigated by testing various combinations of allele specific primers, thereby reducing the number of separate polymerase chain reactions required to type all these alleles.
\end{abstract}

Results-A "three-tube" system worked well and gave accurate results. Tube 1 contained ARMS primers for the detection of IDDM susceptibility alleles DR3 and DR4; tube 2 contained ARMS primers for the detection of susceptibility alleles non-Asp-57 and Arg-52; and tube 3 contained ARMS primers for the detection of the protective alleles Asp-57 and non-Arg-52. DNA samples typed with this ARMS method were in complete agreement with those obtained using the SSO technique.

Conclusion-This method is rapid and has no requirement for radioactivity. It is an efficient method for population screening.

(F Clin Pathol 1993;46:1105-1108)

It is well established that susceptibility to insulin dependent diabetes mellitus (IDDM) is associated with particular HLA class II alleles. Population studies have shown that IDDM is associated with HLA-DR3 and HLA-DR4 alleles, with the risk of developing IDDM particularly increased in DR3 and DR4 heterozygotes. ${ }^{12}$ The HLA DQ genes, which are in linkage disequilibrium with HLA DR, are also strongly associated with IDDM. The presence of DNA sequences coding for an amino acid other than aspartic acid at position 57 (non-Asp-57) of the HLA DQ $\beta$ chain is associated with susceptibility to IDDM, whereas aspartic acid in this position (Asp-57) seems to be related to resistance to the disease. ${ }^{34}$ The DQ $a$ chain alleles bearing arginine in position 52 (Arg-52) have also been reported as conferring susceptibility to IDDM. ${ }^{5}$

The typing of HLA DR3 and DR4, the presence or absence of Asp-57 at the DQ $\beta$ chain, and the presence or absence of Arg-52 at the DQ $a$ chain is typically done by hybridising sequence specific oligonucleotides (SSO) to dot blots of relevant polymerase chain reaction (PCR) products. ${ }^{35}$ The complexity of the HLA system and the large number of HLA alleles associated with IDDM necessitate the use of numerous SSO probes. For example, dot blot analysis for the identification of specific Asp-57 and non-Asp-57 alleles at the DQB1 locus requires nine SSO probes. ${ }^{6}$

Wu et $a l^{7}$ and Newton et $a l^{8}$ have recently described an allele specific PCR (AS-PCR), or the amplification refractory mutation system (ARMS), which is capable of discriminating a single nucleotide change. The principle of ARMS relies on the fact that Taq polymerase, the DNA polymerase commonly used in PCR, lacks a $3^{\prime}$ to $5^{\prime}$ exonuclease activity. Thus a $3^{\prime}$ terminal mismatch of a PCR primer will retard amplification to a significant extent. The extent of the reduction in efficiency of amplification, however, depends on the type of mismatch. ${ }^{78}$ In instances where the mismatch is not sufficiently refractory to extension, a further deliberate mismatch near the $3^{\prime}$ end will often deliver the specificity required. ${ }^{8}$ We used this approach to design allele specific primers for the detection of major susceptibility and protective alleles-DR3, DR4 alleles at the DRB1 locus, Asp-57, non-Asp-57 alleles at the DQB1 locus, and Arg-52, non-Arg-52 alleles at the DQA1 locus in IDDM. We used a multiplex ARMS system ${ }^{9}$ for the detection of these alleles, thereby reducing the number of separate PCR reactions required to type all the alleles. 
Methods

DNA samples used in this study were kindly provided by Dr F Cornelis and were previously DRB1, DQB1, and DQA1 typed using the SSO technique. ${ }^{10-12}$

Oligonucleotide primers were synthesised using an Applied Biosystems 380A DNA synthesizer (Applied Biosystems, Foster City, California, USA). Oligonucleotide primers specific for DR3, DR4, DQ $\beta$ chain Asp-57, non-Asp-57, and DQ $a$ chain Arg-52, nonArg-52 alleles were designed using published sequences. ${ }^{13}$ Primer sequences are shown in the table. Deliberate mismatches to increase primer specificity are underlined. The number in parentheses following each primer indicates the codon number of the last base of the primer. The orientation of the primers (sense or anti-sense) is indicated after each primer.

HLA DR3-specific amplification was directed using DR3 specific sense primer PD3 and anti-sense primer PDR3. The DR3 specific PCR product was 250 base pairs. The DR4 specific amplification was directed using DR4 specific sense primer PD4 and anti-sense primer PDR4. The DR4 specific PCR product was 99 base pairs.

The aspartic acid at position 57 of the DQB1 locus was detected by the use of an ARMS anti-sense primer PSAsp which is specific for alleles containing aspartic acid codon GAC or GAT at position 57. PSAsp was designed such that both Asp-57 alleles containing GAC and GAT at position 57 would be amplified. The sense primer DB130 was previously shown to be specific for the DQB1 and would not amplify the related DQB2 locus. ${ }^{10}$ The Asp-57 specific PCR product was 199 base pairs. The non-Asp-57 allelesthat is, alleles that code for amino acids other than aspartic acid at position 57-were detected by using the sense primer DB130 ${ }^{10}$ and three ARMS anti-sense primers PNAsp1, PNAsp2, and PNAsp3 specific for alleles containing non-aspartic acid codons GCC, GTT, or AGC at position 57. The non-Asp-

Sequence of primers

$D R 3$ specific primers:

PD3: 5' CACGTTTCTTGGAGTAC 3'(10) (sense)

PDR3: 5' CGT AGT TGT GTC TGC AGT AGT TGT CCA ACC 3'(74) (anti-sense)

DR4 Specific primers:

PD4: 5' CAG GTT AAA CAT GAG TGT CAT TTC TTA AAC 3'(19) (sense)

PDR4: 5' GCT GTC GAA GCG CAC GTA CTC CTC TTG GTG 3'(33) (anti-sense) Asp-57 specific primers:

DB130: 5' AGG GAT CCC CGC AGA GGA TTT CGT GTA CC 3'(10) (sense)

PSAsp: 5' TTC CTT CTG GCT GTT CCA GTA CTC GGC CT 3'(57) (anti-sense)

non-Asp-57 specific primers

DB130: as above

PNAsp1: 5' TCC TTC TGG CTG TTC CAG TAC TCG CCG G 3'(57) (anti-sense)

PNAsp2: 5' TCC TTC TGG CTG TTC CAG TAC TCG GAA A 3'(57) (anti-sense)

PNAsp3: 5' TCC TTC TGG CTG TTC CAG TAC TCG GAG C 3'(57) (anti-sense)

Arg-52 specific primers:

PArg1: 5' GAG ACT GTC TGG TGT TTG CCT GTT CTC AGA 3'(52) (sense)

PArg2: 5' GAG ACT GTC TGG CAG TTG CCT CTG TTA CGC 3'(52) (sense)

PCArg3: 5' CTC ATT GGT AGC AGC GGT AGA GTT GTA GCG3'(79) (anti-sense)

PCArg4: 5' CTC ATT GGT AGC AGC GGT AGA GTT GGA GCG 3'(79) (anti-sense)

non-Arg-52 specific primers:

PNArg1: 5' GAG ACT GCC TGG CGG TGG CCT GAG TTA AGC 3'(52) (sense)

PNArg2: 5' GAG ACT GTC TGG AAG TTG CCT CTG TTA CAC 3'(52) (sense)

PCArg 3: as above

PCArg 4: as above

Internal control primers:

AAT-1: 5' CCC ACC TTC CCC TCT CTC CAG GCA AAT GGG 3'

AAT-2: 5' GGG CCT CAG TCC CAA CAT GGC TAA GAG GTG 3

Deliberate mismatches to increase primer specificity are underlined.
57 specific PCR product was 199 base pairs.

The amino acid arginine at position 52 of the DQA1 locus was detected by ARMS sense primers PArg1 and PArg2 which are specific for alleles containing the arginine codons AGA and CGC at position 52 and two anti-sense primers PCArg3 and PCArg4. These primers encompass all sequence variations. The Arg-52 specific PCR product was 138 base pairs. The non-Arg-52 alleles - that is, alleles that code for amino acids other than arginine at position 52 of the DQA1 locuswere detected by using two ARMS sense primers PNArg1 and PNArg2, specific for alleles containing non-arginine codons AGC or CAC at position 52 and two anti-sense primers PCArg3 and PCArg4, which encompass all the sequence variations. The nonArg-52 specific PCR product was 138 base pairs.

Primers AAT-1 and AAT-2, ${ }^{14}$ which amplify a 360 base pair region of exon III of the human $\alpha$-1-antitrypsin gene, were coamplified in all ARMS reactions as an internal positive control.

PCR was carried out as described before ${ }^{15}$ in $100 \mu \mathrm{l}$ reaction volumes with appropriate primers (100 pmol each except for multiplex ARMS where the concentrations of Arg-52 and non-Arg specific primers were $30 \mathrm{pmol}$ each and the concentrations of the AAT internal control primers were 20 pmol each). Genomic DNA $(1 \mu \mathrm{g})$ and 2.5 units of Ampli-Taq DNA polymerase, $100 \mu \mathrm{M}$ each dNTP, 10 mM TRIS-HCl (pH 8.3), $50 \mathrm{mM}$ $\mathrm{KCl}$ (Perkin-Elmer Cetus) were used. $\mathrm{MgCl}_{2}$ was kept at $1.5 \mathrm{mM}$. Thermal cycling was performed using a thermal cycler (PerkinElmer Cetus). The cycling profile for all reactions was $94^{\circ} \mathrm{C}$ for 1 minute, annealing at $57^{\circ} \mathrm{C}$ for 1 minute, and extension at $72^{\circ} \mathrm{C}$ for 1 minute for 30 cycles. The temperature profile above was optimised by testing each set of primers at annealing temperatures ranging from $55^{\circ} \mathrm{C}$ to $60^{\circ} \mathrm{C}$. Seven to ten microlitres of the PCR products were run on $1.5 \%$ agarose gels. Following electrophoresis, the gel was stained with ethidium bromide and photographed under ultraviolet transillumination.

\section{Results}

Allele specificity of PCR primers is conferred by the 3 -terminal nucleotide. ${ }^{7816}$ In some instances additional deliberate mismatches near the $3^{\prime}$ end of certain primers were required to provide the necessary specificity. To test the allele specificity of the primers constructed to detect DR3, DR4, Asp-57, non-Asp-57, Arg-52 and non-Arg-52 alleles, each set of primers was tested separately using DNA samples from 15 individuals previously typed for DRB1, DQB1, and DQA1 using the SSO technique. ${ }^{10-12}$ The results were in complete concordance with those obtained by SSO analysis. Figure 1 shows the representative data on four DNA samples illustrating the specificity of the ARMS primers. 
DR3

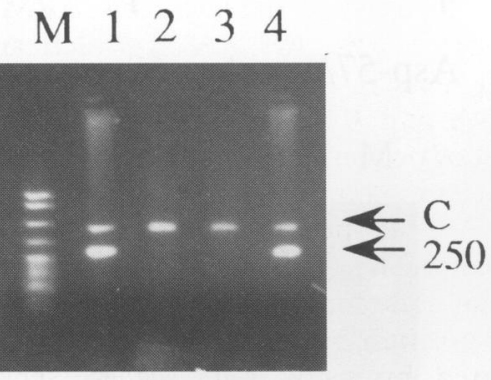

Asp-57

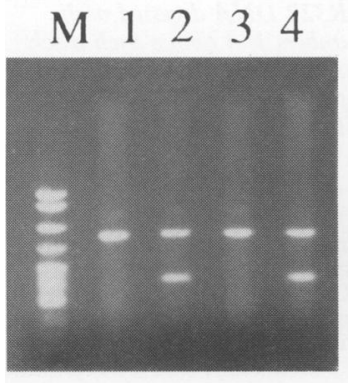

Arg-52

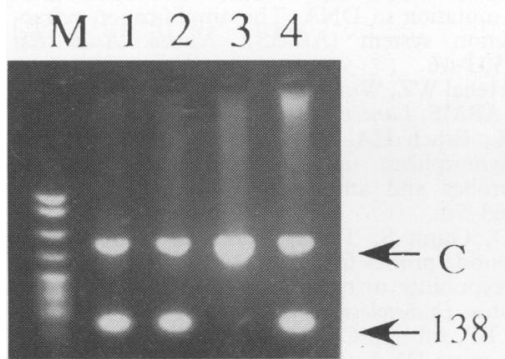

DR4

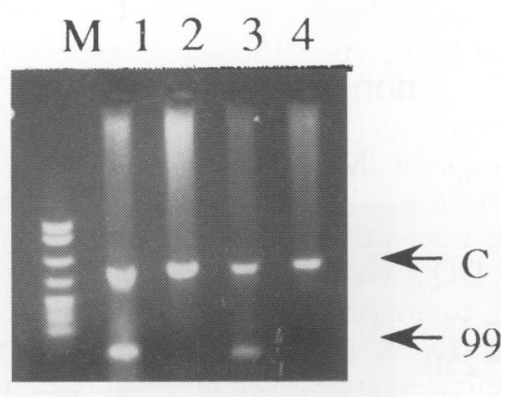

non-Asp-57

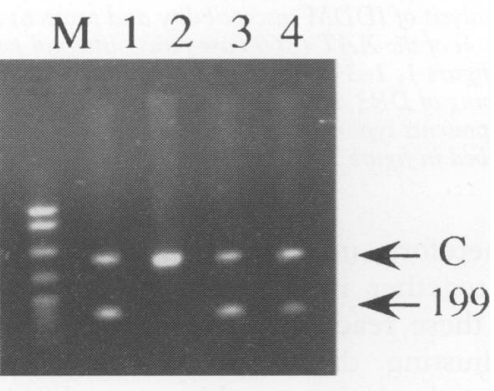

non-Arg-52

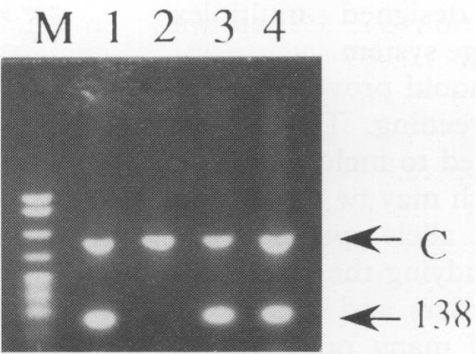

Figure 1 ARMS analysis of IDDM susceptibility and protective alleles. $M$ is a pBR322 $D N A$ digested with MspI (marker). C denotes the position of the AAT ( 360 base pairs) internal positive control. 250, 99 indicate the sizes in base pairs of the DR3 and DR4 specific PCR products, respectively. 199 indicates the size in base pairs of the Asp-57 and non-Asp-57-specific products and 138 indicates the size in base pairs of the Arg-52 and non-Arg-52-specific products. Numbers $1-4$ above each track represent DNA from individuals previously typed by SSO technique. Case 1: DRB1*0301/0404 (DR3/DR4); $D Q B 1^{*} 0201 / 0302$ (non-Asp-57/non-Asp-57); DQA1*0301/0501 (non-Arg-52/ Arg-52). Case 2: DRB1*1101/1101 (DRX/DRX); DQB1*0301/0301 (Asp57/Asp57); $D Q A 1^{*} 0501 / 0501$ (Arg-52/Arg-52). Case 3: DRB1*0401/07

(DR4/DRX);DQB1*0201/0302 (non-Asp-57/non-Asp-57); DQA1*0201/0301 (non-Arg-52/non-Arg-52). Case 4: DRB1*0301/1401 (DR3/DRX); DQB1*0201/0503 (non-Asp-57/Asp-57); DQA1*0101/0501 (non-Arg-52/Arg-52). DRX denotes any allele other than DR3 or DR4. were typed in tube 1 containing the primers PDR3, PD3, PDR4 and PD4.

Susceptibility alleles non-Asp-57 and Arg52 were typed in tube 2 containing the primers DB130, PNAsp1, PNAsp2, PNAsp3, PArg1, PArg2, PCArg3 and PCArg4. The IDDM protective alleles Asp-57 and nonArg-52 were typed in tube 3 containing the primers DB130, PSAsp, PNArg1, PNArg2, PCArg3 and PCArg4 (fig 2, table). The same 15 DNA samples as above were typed using this strategy and the results were in complete agreement with those obtained by typing the alleles separately and with those obtained by the SSO technique. Figure 2 shows the representative data on four DNA samples.

\section{Discussion}

There is increasing interest in typing individuals and population groups for HLA alleles which are related to susceptibility to IDDM. In this study we have shown that it is possible to type for several major susceptibility and protective HLA alleles for IDDM by ARMS. These include the DR3 and DR4 at the DRB1 locus, Asp-57 and non-Asp-57 at the DQB1 locus, and Arg-52 and non-Arg-52 at the DQA1 locus. To type individuals for all these alleles using the standard SSO technique would be time consuming because of the need for dot blots and the large number of SSO probes each with its own washing condition. In contrast, the ARMS method for the detection of these alleles has the advantage of being rapid and non-radioactive.

The efficiency of the ARMS system may be further enhanced in a multiplex format. Thus we are able to type all common IDDM related HLA alleles using a simple three-tube assay. Compared with conventional multiplex PCR, ${ }^{17}$ multiplex ARMS requires more careful primer design and more stringent experimental conditions. Similar to conventional multiplex PCR, the ARMS products should be distinguishable from each other by length and by the fact that the primers should work together at a certain annealing temperature. This latter requirement may need some experimentation to achieve, because allele specificity is also temperature dependent. The primers should not interact to form spurious products. The design of relatively long primers (28-30 bases) with about $50 \%$ GC content permits the use of higher annealing temperature, thus minimising spurious product formation. Furthermore, as the primers are designed to bind to adjacent polymorphic regions of an HLA gene, they may form unwanted, but nevertheless, legitimate PCR products in combination. To circumvent this, as in the DR3 and DR4 systems, we used a double ARMS format ${ }^{18}{ }^{19}$ in which both the $5^{\prime}$ and $3^{\prime}$ primers are allele specific and product formation by the $5^{\prime}$ primer to one allele with the $3^{\prime}$ primer to another allele is not possible. Finally, different primers have different amplification efficiencies-for example, the ARMS primers for Arg-52 consistently produce more PCR product than the of ARMS primers and found that the PCR reactions divided into three sets worked well and gave accurate results. Hence a "threetube" test strategy was subsequently used. IDDM susceptibility alleles DR3 and DR4 


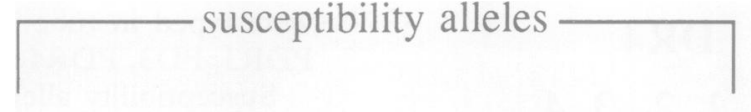

DR3/DR4

$\begin{array}{lllll}M & 1 & 2 & 3 & 4\end{array}$

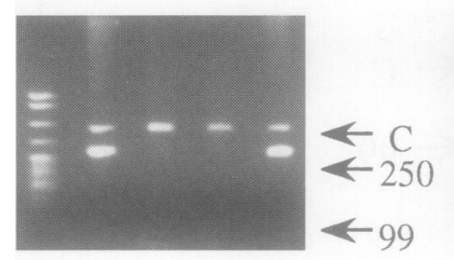

non-Asp-57/Arg-52

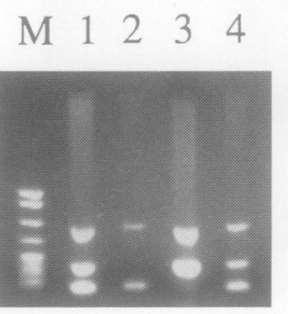

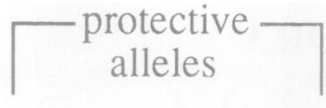

Asp-57/non-Arg-52

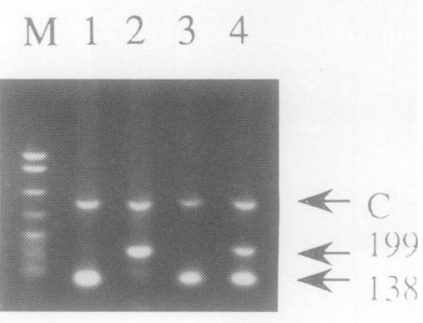

2

Figure 2 Multiplex ARMS analysis of IDDM susceptibility and protective alleles. $M$ is $p B R 322$ DNA digested with MspI (marker). C denotes position of the AAT (360 base pairs) internal positive control. Numbers $1-4$ above each track represent the same four cases as figure 1.1-3 under the gels represent the three sets of multiplex PCR necessary for full typing. Tube 1 represents the typing of DR3 and DR4-specific alleles; tube 2 represents the typing of non-Asp-57 and Arg-52-specific alleles; tube 3 represents typing of Asp-57 and non-Arg-52-specific alleles. 250, 99, 199 and 138 denote the same ARMS products described in figure 1.

non-Asp-57 primers. Therefore, in order for these primers to work together in a single tube, the efficiencies of these reactions have to be balanced by adjusting the relative primer ratio. Other potential solutions to this problem include varying the type of extra mismatch or the length of primers so as to match their amplification efficiency. Nevertheless, a well designed multiplex ARMS is a rapid one-stage system.

This new approach should prove very efficient for population screening. The system can also easily be modified to include further susceptibility alleles which may be discovered in the future. Multiplex allele specific PCR should be of value in studying the HLA susceptibility to other disorders and indeed for the rapid genotyping of many other multiallelic systems.

We thank Dr RC Turner for helpful discussion. We are also grateful to Drs F Cornelis and S Bains for DNA samples. This work was supported by the Medical Research Council (UK) and the Alan and Babette Sainsbury Trust. YMDL is a Wellcome Medical Graduate Fellow.

1 Owerbach D, Lernmark A, Platz P, et al. HLA-D region $\beta$-chain DNA endonuclease fragments differ between HLA-DR identical healthy and insulin-dependent individuals. Nature 1983;303:815-7.

2 Thomson G, Robinson WP, Kuhner MK, et al. Genetic heterogeneity, modes of inheritance and risk estimates for a joint study of caucasians with insulin-dependent for a joint study of caucasians with insulin-dependent

3 Todd JA, Bell JI, McDevitt HO. HLA-DQ beta gene contributes to susceptibility and resistance to insulin dependent diabetes mellitus. Nature 1987;329:599-603.

4 Morel PA, Dorman JS, Todd JA, McDevitt HO, Trucco $M$. Aspartic acid at position 57 of the HLA-DQ $\beta$ chain protects against type 1 diabetes: a family study. Proc Natl Acad Sci USA 1988;85:8111-5.

5 Khalil I, D'Auriol L, Gobet $M$, et al. A combination of HLA-DQB Asp57-negative and HLA DQA Arg52 confers susceptibility to insulin-dependent diabetes mellitus. F Clin Invest 1990;85:1315-9.

6 Dorman JS, LaPorte RE, Stone RA, Trucco $M$ Worldwide differences in the incidence of type 1 diabetes are associated with amino acid variation at position 57 of the HLA-DQ beta chain. Proc Natl Acad Sci tion 57 of the HLA-D
USA $1990 ; 87: 7370-4$.

7 Wu DY, Ugozzoli L, Pal BK, Wallace RB. Allele-specific enzymatic amplification of $\beta$-globin genomic DNA for diagnosis of sickle cell anemia. Proc Natl Acad Sci USA 1989;86:2759-60.

8 Newton CR, Graham A, Heptinstall LE, et al. Analysis of any point mutation in DNA. The amplification refractory mutation system (ARMS). Nucleic Acids Res 1989;17:2503-16.

9 Lo Y-MD, Mehal WZ, Wordsworth BP, et al. HLA typing by double ARMS. Lancet 1991;338:65-6.

10 Bugawan TL, Erlich HA. Rapid typing of HLA-DQB1 DNA polymorphism using nonradioactive oligonucleotide probes and amplified DNA. Immunogenetics 1991;33:163-70.

11 Owerbach D, Gunn S, Ty G, Wible L, Gabbay KH Oligonucleotide probes for HLA-DQA and DQB genes define susceptibility to type I (insulin-dependent) diabetes mellitus. Diabetologia 1988;31:751-7.

12 Wordsworth BP, Allsopp CEM, Young RP, Bell JI. HLADR typing using DNA amplification by the polymerase chain reaction and sequential hybridization to sequencespecific oligonucleotide probes. Immunogenetics 1990;32: 413-8.

13 Marsh SGE, Bodmer JG. HLA class II nucleotide sequences, 1991. Immunogenetics 1991;33:321-34.

14 Newton CR, Kalsheker N, Graham A, et al. Diagnosis of $\alpha_{1}$-antitrypsin deficiency by enzymatic amplification of $\alpha_{1}$-antitrypsin deficiency by enzymatic amplification of
human genomic DNA and direct sequencing of polyhuman genomic DNA and direct sequencing of polymerase chain

15 Saiki RK, Gelfand DH, Stoffel S, et al. Primer-directed enzymatic amplification of DNA using a thermostable DNA polymerase. Science 1989;239:487-91.

16 Kwok S, Kellogg DE, McKinney N, et al. Effects of primer-template mismatches on the polymerase chain reaction: human immunodeficiency virus type 1 model studies. Nucleic Acids Res 1990;18:999-1005.

17 Chamberlain JS, Gibbs RA, Ranier JE, Nguyen PN, Caskey CT. Deletion screening of the Duchenne muscular dystrophy locus via multiplex DNA amplification. cular dystrophy locus via multiplex

18 Lo Y-MD, Patel P, Newton CR, et al. Direct haplotype determination by double ARMS: specificity, sensitivity and genetic applications. Nucleic Acids Res 1991;19: 3561-7.

19 Lo Y-MD, Patel P, Mehal WZ, Fleming KA, Bell J, Wainscoat JS. Analysis of complex genetic systems by ARMS-SSCP: applications to HLA genotyping. Nucleic Acids Res 1992;20:1005-9. 